\title{
CAPÍTULO 43: ESTRATÉGIAS PARA O ARMAZENAMENTO E MANUTENÇÃO DE QUALIDADE DA NOZ-PECÃ APÓS A COLHEITA
}

\section{CHAPTER 43: STRATEGIES FOR STORAGE AND MAINTENANCE OF PECAN NUTS QUALITY AFTER HARVEST}

\author{
Stephanie Reis Ribeiro ${ }^{1}$; Vanderlei Both ${ }^{2}$; Roger Wagner $^{3}$
}

\begin{abstract}
Resumo
A noz-pecã é um fruto seco rico em compostos nutritivos, considerado um alimento promotor de saúde. No entanto, a manutenção da qualidade da noz-pecã no período pós-colheita torna-se um desafio, devido ao seu alto teor lipídico, constituído majoritariamente de ácidos graxos insaturados suscetíveis à oxidação. Nesse processo pode ocorrer escurecimento das nozes, perda da crocância, formação de odores rançosos, bem como a redução das propriedades nutricionais, comprometendo a integridade e, consequentemente, perdas na comercialização. Além disso, o beneficiamento ineficiente também impacta no desenvolvimento de microrganismos, especialmente os fungos, que além de acelerar as reações de degradação podem produzir micotoxinas, as quais são nocivas à saúde do consumidor. Apesar de sua importância nutricional e econômica, há poucas informações sobre condições ideais de armazenamento e vida-de-prateleira das nozes-pecã. Em geral, temperaturas abaixo de $10{ }^{\circ} \mathrm{C}$, teor de umidade em torno de 4\%, umidade relativa de 40-60\%, concentração de oxigênio menor que $3 \mathrm{kPa}$ de $\mathrm{O}_{2}$ são recomendadas para o armazenamento de nozes. Esta revisão foi realizada para congregar diferentes técnicas e parâmetros de armazenamento (temperatura, tempo, umidade, etc.), bem como os tipos de embalagens na estocagem, visando a manutenção da qualidade das nozes-pecã. Dessa forma, o conhecimento sobre as condições recomendadas para o armazenamento e beneficiamento da noz-pecã favorece a conservação dos atributos nutricionais e sensoriais.
\end{abstract}

Palavras-Chave: Carya illinoinensis, armazenamento, conservação, qualidade.

\begin{abstract}
Pecan is a dry fruit rich in nutritious compounds, considered a health-promoting food. However, maintaining pecan quality in the post-harvest period becomes a challenge, due to its high lipid content, mostly composed of unsaturated fatty acids susceptible to oxidation. In this process, darkening of the nuts, loss of crispness, formation of rancid odors may occur, as well as the reduction of nutritional properties, compromising integrity and, consequently, losses in commercialization. In addition, inefficient processing also impacts the development of microorganisms, especially fungi, which in addition to accelerating degradation reactions can produce mycotoxins, which are harmful to consumer health. Despite its nutritional and economic importance, there is little information on ideal storage conditions and shelf-life for pecans. In general, temperatures below $10{ }^{\circ} \mathrm{C}$, moisture content around $4 \%$, relative humidity $40-60 \%$, oxygen concentration less than $3 \mathrm{kPa}$ of $\mathrm{O}_{2}$ are recommended for the storage of nuts. This review was carried out to gather different techniques and storage parameters (temperature, time, humidity, etc.), as well as the types of packaging in storage, with a view to maintaining the quality of pecans. Thus, knowledge about the recommended conditions for the storage and

\footnotetext{
${ }^{1}$ Doutoranda, Ciência e Tecnologia dos Alimentos, Universidade Federal de Santa Maria, stephanieribeiro18@hotmail.com

${ }^{2}$ Professor, Doutor, Agronomia, Universidade Federal de Santa Maria, vanderleiboth@yahoo.com.br

${ }^{3}$ Professor, Doutor, Ciência e Tecnologia dos Alimentos, Universidade Federal de Santa Maria, rogerwag@gmail.com
} 
RIBEIRO, S. R.; BOTH, V. e WAGNER, R.

processing of pecans favors the conservation of nutritional and sensory attributes.

Keywords: Carya illinoinensis, storage, conservation, quality.

\section{Introdução}

O incentivo ao consumo da noz-pecã [Carya illinoinensis (Wangenh.) K. Koch] alia o sabor característico e muito apreciado pelo consumidor, as diferentes formas de apresentação (in natura, cozidas, caramelizada, salgadas, em pasta, óleo, etc.) (KING et al., 2008) e os benefícios à saúde (SALVADOR et al. 2016). Dessa forma, a noz-pecã é considerada um alimento de alto valor agregado e detém um mercado promissor para os investidores (Fronza et al., 2015). A noz-pecã é produzida em maior escala nos EUA (ERTEN, 2016), com cerca de 55\% da produção mundial (FRONZA et al. 2018), (aproximadamente 127.000 ton). O Brasil apresenta atualmente uma produção próxima de 3.500 t em 2019, colocando o país entre os quatro maiores produtores do mundo, atrás apenas dos EUA, México e África do Sul (ABNC, 2019). A produção nacional de pecãs está concentrada na Região Sul, sendo estado do Rio Grande do Sul o responsável por aproximadamente 50\% da produção nacional (LESINA, 2020; SILVA, 2017). Nesta região percebe-se um incremento significativo na implantação de novas áreas de nogueira pecã. Como a cultura demora cerca de seis anos para iniciar a produção de frutos, a expectativa é que haja um grande aumento da produção nos próximos anos.

A noz-pecã é classificada como um fruto seco altamente lipídico (cerca de 50\% a 70\%) (SALVADOR et al., 2016; VENKATACHALAM, 2006), mas também é uma excelente fonte de outros nutrientes, como proteínas e fibras (10 e 14\%, respectivamente) (RIBEIRO et al., 2020). Contudo, o elevado teor de lipídeos, apresenta predominantemente os ácidos graxos insaturados (AGI), como os ácidos graxos oleico, linoleico e linolênico (SALVADOR et al., 2016; ZHAO, 2011). Esses ácidos graxos, embora possuam efeitos benéficos reconhecidos à saúde humana (KING et al., 2008; TORABIAN et al., 2009), tornam a noz suscetível à deterioração por hidrólise e subsequente oxidação (MASKAN; KARATAS, 1998). As reações de deterioração lipídica estão entre os principais fatores que depreciam as nozes, formando compostos que atribuem descritores sensoriais relacionados ao ranço (off-flavors), promovendo também o escurecimento da mesma (RIBEIRO, et al., 2020; IVANOVA-PETROPULOS, et al., 2015). Nesse sentido, ainda não há legislação que ampare os atributos de qualidade da nozpecã, mas leva-se em consideração os parâmetros químicos para óleo de oliva bruto, utilizado pela ANVISA (BRASIL, 2005).

O aumento significativo da área cultivada de nogueira-pecã demanda estudos que busquem auxiliar os produtores no processamento pós-colheita, pré-armazenamento e 
RIBEIRO, S. R.; BOTH, V. e WAGNER, R.

armazenamento (MOKOCHINSKI, 2015). São escassos os estudos sobre armazenamento da noz-pecã. Em estudo recente foi observado boa manutenção da qualidade, após 12 meses, com a armazenagem de nozes em uma condição de umidade relativa do ambiente menor do que $60 \%$, temperaturas inferiores a $10^{\circ} \mathrm{C}$ e disponibilidade de $\mathrm{O}_{2}$ abaixo de $3 \mathrm{kPa}$ (RIBEIRO et al., 2020) (Tabela 1). No entanto, muitos produtores inda realizam o armazenamento em silos ou containers, em que as nozes estão submetidas a temperatura e umidade relativa ambiente após a devida secagem, sujeitas às alterações fisiológicas com perdas na qualidade (MA et al., 2013; MA et al., 2010) e suscetíveis ao crescimento fúngico. Os limites aceitáveis para os parâmetros de qualidade no prazo de validade das nozes tem base nos grãos quando percebidos como não comestíveis (escuras e com sabor rançoso) (PANKAEW et al., 2016).

Várias técnicas já foram aplicadas no armazenamento de nozes e grãos armazenados para retardar sua deterioração, como o uso de substâncias químicas por fumigação (BOUKAEW et al., 2017), tratamento com plasma atmosférico frio (SOHBATZADEH et al., 2016), irradiação (ALGABR et al., 2013) e dióxido de cloro (LEE et al., 2013). Porém, ainda são incipientes os trabalhos voltados a estudar as condições de armazenamento de pecãs (RIBEIRO et al., 2020). Dessa forma, existe uma lacuna a ser explorada visando amenizar as reações de oxidação e crescimento fúngico ao longo do período pós-colheita da noz-pecã. Sendo assim, a presente revisão visa abordar diferentes métodos e condições de armazenamento, incluindo o emprego de câmaras com atmosfera controlada, bem como fornecer informações sobre os efeitos do armazenamento na qualidade das nozes-pecã.

\section{Cultivo e consumo da noz-pecã}

Nativa do sudeste dos Estados Unidos e México (SANTERRE, 1994), o cultivo da nogueira-pecã se estendeu a vários outros países no início do século XX (China, Austrália, África do Sul, Israel, Argentina e Brasil), os quais respondem pelo restante da produção comercial (FRONZA, et al. 2018). No Brasil são cultivados principalmente os seguintes tipos de nozes: castanha-do-Brasil, castanha-de-caju, macadâmia e pecã. Dentre elas, a noz-pecã tem sua maior produção no estado do Rio Grande do Sul, com cerca de 50\% (LESINA, 2020). A pecanicultura nesse estado tem proporcionado uma importante fonte de renda tanto para pequenas como em grandes propriedades (FRONZA et al., 2015).

A noz-pecã presenta versatilidade em suas apresentações, podendo ser consumidas in natura ou cozidas, caramelizada, salgadas, granuladas, em pasta e, também na forma de óleo culinário, que pode ser obtido por prensagem mecânica, sendo considerado uma especiaria muito apreciada na Europa (SALVADOR, 2014; King et al., 2008). Além de tudo, o óleo 
RIBEIRO, S. R.; BOTH, V. e WAGNER, R.

também é um componente de cosméticos, loções, sabões, perfumes e óleos para massagem (SAHENA, 2009; VILLARREAL-LOZOYA, 2007). Entretanto, é uma das nozes mais saborosas para ser consumida in natura.

\section{Composição química e parâmetros de qualidade}

Classificados como fruto seco (MURDOCK, 2002), as nozes-pecã são fontes de lipídeos ricos em AGI (em torno de 90\%) (SALVADOR et al., 2016). Os principais ácidos graxos encontrados são o oleico (C18:1 n9), linoleico (C18:2 n6) e linolênico (C18:3 n3), nas concentrações aproximadas de 63\%, 18\% e 3\%, respectivamente (SALVADOR et al., 2016; ZHAO, 2011). Além dos lipídeos, são importantes fontes de outros macronutrientes, podendo apresentar na sua composição química cerca de 9,9 g de proteínas; 7,8 g de carboidratos; 3,7 g de umidade; 1,4 g de cinzas (minerais totais) a cada $100 \mathrm{~g}$ de noz (ORO et al., 2008; Venkatachalam, 2006). Os elementos cálcio, fósforo e ferro, além das vitaminas B2 e A foram reportadas por Rainey e De Nyquist (1997). Adicionalmente, apresenta expressivas concentrações de fitoesterois totais em sua composição (1000 a $2900 \mathrm{mg} \mathrm{kg}^{-1}$ ), bem como tocoferóis $\left(\alpha, \gamma\right.$ e $\delta$ ), podendo chegar a $370 \mathrm{mg} \mathrm{kg}^{-1}$ (THOMPSON; KADER, 2012; WCRF, 2007).

Alguns atributos de qualidade, como cor, aroma, crocância, ausência de contaminação devem ser observados para o consumo da noz-pecã, seja ela in natura ou qualquer forma de processamento, (WOODROOF, 1967). Na comercialização, há classificações de acordo com a massa da amêndoa, o tamanho e as características da casca, que são utilizadas para definição de preços no mercado (CARGNELUTTI FILHO et al., 2014). A qualidade das amêndoas é obtida pelo manejo adequado realizado no campo durante a estação de cultivo. Essa qualidade deve ser mantida após a colheita pela estocagem em condições adequadas de armazenamento. Dessa forma, a vida-de-prateleira das nozes é afetada por diferentes fatores inerentes ao cultivar, manejo no campo, condições edafoclimáticas, bem como de técnicas de colheita, secagem, armazenamento e processamento, quando for o caso.

As nozes tendem a desenvolver rancidez rapidamente se armazenadas em condições inadequadas. Sendo assim, o índice de acidez e o índice de peróxidos estão entre os principais parâmetros químicos avaliados para qualidade de nozes (RIBEIRO et al., 2020; WALTON et al., 2017; GECGEL et al., 2011) (Tabela 1). O índice de acidez (expressa a porcentagem de ácidos graxos livres) esta relacionada com o desenvolvimento de reações hidrolíticas no óleo e comumente expressos os ácidos graxos livres da noz em miligrama equivalentes de ácido oleico por grama de óleo (ROSSELL, 1986). Através da RDC No 270 (2005) a ANVISA determina 
como parâmetro de qualidade para óleos brutos uma acidez máxima de 1,0 mg de ácido oleico $\mathrm{g}^{-1}$ de óleo, enquanto que determina para óleos refinados o máximo de $0,3 \mathrm{mg}$ ácido oleico $\mathrm{g}^{-1}$ de óleo (BRASIL, 2005). A medida do índice de peróxidos é utilizada como um indicador dos estágios iniciais de oxidação lipídica. Na RDC No 270 (2005) é estipulado os valores de peróxidos em óleos vegetais de no máximo 15 meq de $\mathrm{O}_{2} \mathrm{~kg}^{-1}$ de óleo, e para refinados de no máximo 5 meq de $\mathrm{O}_{2} \mathrm{~kg}^{-1}$ de óleo.

\section{Manutenção da qualidade no armazenamento de nozes}

A exposição das nozes a níveis elevados de oxigênio, temperatura, umidade e entre outros fatores como a presença de casca, película protetora e eventuais quebras podem influenciar na velocidade das reações oxidativas (RIBEIRO et al., 2020) e crescimento microbiano (GUADAGNI et al., 1978). Logo, para reduzir o desenvolvimento de características indesejáveis (escurecimento, rancidez, etc.), diferentes técnicas e materiais de embalagem, (GARCÍA-PASCUAL et al., 2003) tem sido pesquisadas e/ou utilizadas para aumentar a vidade-prateleira das nozes (Tabela 1).

\section{Secagem}

As nozes-pecã normalmente são colhidas pela ocasião da abertura do epicarpo (shunck). Pode ser realizada com equipamentos mecanizados que provocam vibrações nas árvores para a queda das nozes (shaker), ou manualmente logo após a queda. É importante a coleta imediata par evitar contaminações pelo solo. Neste momento, as nozes apresentam elevado teor de umidade, o que acelera as reações hidrolíticas e oxidativas (GAMA et al., 2018), crescimento de fungos, aumento da germinação e, consequentemente, redução no prazo de validade. Portanto, essa umidade excessiva precisa ser removida o mais rápido possível. Para uma secagem eficiente é indispensável o conhecimento sobre as características das nozes, de forma a otimizar nas operações de secagem a temperatura e o tempo, bem como o desenho ideal dos secadores (PANKAEW et al., 2016), podendo ser natural, em fornos ou microondas (SILVA et al., 2016).

O teor de umidade está correlacionado com a atividade da água (aw). Esta é uma medida que ser refere a água livre, que pode influenciar na velocidade das reações físicas, químicas, enzimáticas (MANEFFA et al., 2017) e metabólicas (SABLANI et al., 2007), estando diretamente relacionada ao tempo de vida-de-prateleira. Além disso, elevados valores de aw favorecem o desenvolvimento de bolores entre outros microorganismos durante $o$ armazenamento nas nozes (SILVA et al., 2016; VENKATACHALAM; SATHE, 2006). Em geral pecãs apresentam valores de aw entre 0,6 a 0,8 (VENKATACHALAM, 2006). Entretanto, 
RIBEIRO, S. R.; BOTH, V. e WAGNER, R.

quando se considera o tempo de armazenamento em temperatura ambiente $\left(\sim 20^{\circ} \mathrm{C}\right.$ a $\left.25^{\circ} \mathrm{C}\right)$, as pecãs com aw de $\sim 0,75$ desenvolvem bolores após 16 semanas, enquanto que as armazenadas com aw de 0,9 desenvolvem bolores em apenas 4 semanas de armazenamento (VENKATACHALAM; SATHE, 2006).

Em relação aos aspectos sensoriais, a secagem ineficiente ou não realizada, causa diversos fenômenos indesejáveis (desenvolvimento fúngico, deterioração por reações hidrolíticas) e, consequentemente, prejuízos na comercialização. A noz macadâmia em casca, com alto teor de umidade, desenvolve coloração marrom nas amêndoas, reduzindo a sua vida útil (PHATANAYINDEE et al., 2012; WALTON et al., 2013). Portanto, essa classe de alimentos requer uma secagem o mais rápido possível para minimizar as perdas de qualidade (PANKAEW et al. 2016). Pistaches com 4\% de umidade apresentaram maior luminosidade (coloração mais clara), firmeza, e doçura do que pistaches com $11 \%$ de umidade, as quais apresentam valores quatro vezes maiores nos parâmetros de rancidez e amargor. Ademais, as pistaches mais secas (4\%) mantiveram sua qualidade preservada por 12 meses, quando armazenadas a $20{ }^{\circ} \mathrm{C}$ (KADER et al., 1982). Dessa forma, a partir de uma secagem bem sucedida as nozes tendem a manter os aspectos de qualidade e estender sua vida útil. Além desse ponto, existem outros fatores positivos com a aplicação do processo de secagem nas nozes, como a facilitação do descascamento e redução de amêndoas de castanhas de caju quebradas, devido ao teor de umidade mais baixo (OGUNSINA; BAMGBOYE, 2012).

\section{Temperatura de armazenamento}

A temperatura é um fator que influencia diretamente nas reações químicas, enzimáticas e crescimento microbiano, bem como pode modificar a composição química, através da alteração do teor de água (RIBEIRO et al., 2020; ARENA et al., 2013; LEUFVEN et al., 2010). $\mathrm{O}$ armazenamento de nozes em temperaturas baixas $\left(0\right.$ a $\left.7{ }^{\circ} \mathrm{C}\right)$ prolonga a vida-de-prateleira das nozes (CHRISTOPOULOS; TSANTILI, 2015), retardando a evolução dos principais indicadores de perda de qualidade, como aumento de índice de acidez e peróxidos (RIBEIRO et al., 2020). Estes autores realizaram um experimento em que armazenaram por 12 meses nozes-pecã com casca, da cultivar Barton, em diferentes temperaturas $\left(1,5,10\right.$ e $\left.20^{\circ} \mathrm{C}\right)$. Os resultados encontrados indicam que as temperaturas mais baixas apresentaram maior luminosidade $\left(\mathrm{L}^{*}\right)$, coloração amarelo/dourada $\left(\mathrm{b}^{*}\right)$ e menor índice de acidez, enquanto as nozes estocadas a $20^{\circ} \mathrm{C}$ apresentaram maiores índices de peróxidos e coloração avermelhada (a*). Da mesma forma, amêndoas (Prunus dulcis, cultivar Mamaei) inteiras armazenadas a 4 ${ }^{\circ} \mathrm{C}$ apresentaram índice de peróxidos e trienos conjugados menores em relação às armazenadas 
RIBEIRO, S. R.; BOTH, V. e WAGNER, R.

a $23{ }^{\circ} \mathrm{C}$. Adicionalmente, as amêndoas estocadas a $4{ }^{\circ} \mathrm{C}$ apresentaram maiores escores sensoriais para os atributos cor e odor ao final de 10 meses.

A redução da temperatura tem se mostrado um fator efetivo no retardo da ação de lipoxigenases (GAMA et al., 2018) e oxidação dos lipídeos, que desencadeiam a produção de hidroperóxidos (GAMLI; HAYOĞLU, 2007; LEUFVEN et al., 2010) e aumento dos ácidos graxos livres (WALTON et al., 2017). Em decorrência dessas reações de deterioração dos ácidos graxos observa-se a formação de compostos voláteis relacionados ao aroma e sabor de ranço (aldeídos, cetonas, álcoois) (RIBEIRO et al., 2020; MEXIS; KONTOMINAS, 2009b), em especial o aldeído hexanal (JENSEN et al., 2001, 2003; MEXIS; KONTOMINAS, 2009a).

\section{Controle de luminosidade}

As reações catalisadas pela luz podem prejudicar a qualidade e a estabilidade de alimentos com alto teor de óleos e gorduras (SATTAR, 1989), podendo ser pela oxidação lipídica ou de degradação das vitaminas presentes (MIN; BOFF, 2002). Nozes armazenadas em condições com maior luminosidade têm valores mais altos de peróxido, concentração de hexanal, sabor rançoso e amargo, bem como alterações na cor, em comparação com nozes armazenadas no escuro (MEXIS et al., 2009), devido oxidação de pigmentos e ácidos graxos (RAEI et al., 2009). Em óleos extraídos de nozes a taxa de peroxidação é profundamente diminuída pelo armazenamento em vidro âmbar ou eliminação completa da luz (SATTAR, 1989). O óleo de noz chilena (Walnut) apresentou valores de peróxidos de 1,57 quando exposto à luz, 0,15 no escuro, 1,20 em recipiente transparente e 0,19 meq de $\mathrm{O}_{2} \mathrm{~kg}^{-1}$ de óleo quando em vidro ambar (SATTAR, 1989). Na avaliação de óleo de amêndoas, armazenados em temperatura ambiente durante o período de 2 meses, os valores de ácidos graxos livres obtidos, quando expostos a luz e no escuro, respectivamente, foram de $0,59 \%$ e $0,49 \%$, da mesma forma, os valores médios de peróxido foram 25.67 e 19.15 meq de $\mathrm{O}_{2} \mathrm{~kg}^{-1}$ de óleo (ATSU-BARKU et al., 2012). Portanto, o controle da luminosidade no armazenamento de nozes, também nos óleos extraídos, é importante para minimizar as reações induzidas pela luz.

\section{Radiação}

As radiações ultravioleta, raios gama, feixe de elétrons e microondas são comumente utilizadas em estudos da manutenção de qualidade de nozes (SILVA et al., 2016; KARAGÖZ et al., 2014; BARREIRA et al., 2012; DE MELLO, SCUSSEL, 2009). O uso de radiações é uma alternativa para substituir tratamentos tradicionais como a fumigação, as quais podem contaminar nozes e o ambiente com os resíduos químicos (KUME et al., 2009). No entanto, a aplicação das doses de irradiação devem ser otimizadas, visando a máxima eficiência na 
redução microbiana, eliminando os riscos biológicos, com a mínima modificação química e sensorial das nozes (MA et al., 2013; BARREIRA et al., 2012). A irradiação por micro-ondas pode ser utilizada tanto na secagem, como para o controle de fungos deterioradores e produtores de micotoxinas (SILVA et al., 2016; FANG et al., 2011; LUTER et al., 1982). Na secagem de castanhas do Pará, o uso do micro-ondas apresentou desempenho adequado, diminuindo em até 43,5\% no teor de umidade e, consequentemente na redução da aw (SILVA et al., 2016). Em outro estudo, mostrou a diminuição da contagem de Aspergillus parasiticus (FANG et al., 2011).

O uso de diferentes níveis de radiação gama (1, 3, 5, and 7 kGy) em avelã, walnut, amêndoa doce e pistache não alteraram significativamente o teor de óleo das nozes, no entanto, o teor de ácidos graxos livres e índice de peróxidos aumentaram proporcionalmente à dose. Além disso, a concentração de ácidos graxos saturados totais aumentou enquanto os ácidos graxos monoinsaturados e poliinsaturados totais diminuíram com doses maiores de radiação (GECGEL et al., 2011). Em pecãs inoculadas com Escherichia coli e Salmonella Typhimurium, nas ranhuras dorsais ou na superfície plana, e irradiadas à temperatura ambiente usando um acelerador de feixe eletrônico de 1,35 MeV a 0,2, 0,4, 0,6 e 0,8 kGy em embalagem a vácuo, embalagem a ar, $100 \%$ de embalagem de nitrogênio e $100 \%$ de embalagem de oxigênio, não houve diferenças ( $\mathrm{p}>0,05)$ nos valores de D10 (dose necessária para a descontaminação) entre os microrganismos nas diferentes atmosferas. Em contrapartida, a irradiação sob atmosfera de nitrogênio mostrou-se como alternativa promissora, pois retarda o aparecimento de ranço, quando comparada à irradiação sob condições atmosféricas ou 100\% de oxigênio.

\section{Embalagens}

As embalagens são essenciais para contenção e apresentação dos alimentos, além de contribuir para minimizar reações que afetam a sua integridade. A qualidade e a vida útil dos alimentos são influenciadas pelas propriedades de barreira da embalagem contra umidade, oxigênio e a interação de constituintes do alimento com o material (ORO, 2007). Diversos materiais podem ser utilizados, como o plásticos e alumínio, tanto com ou sem selamento a vácuo (RAISI, et al., 2015; MEXIS et al., 2009) (Tabela 1). Ribeiro et al., (1993) estudaram a estabilidade de castanhas-do-pará descascadas em bandejas de polipropileno (PP) cobertas com filmes de cloreto de polivinila (PVC), quando estocadas à temperatura ambiente por 12 meses. Nesse trabalho os autores reportaram que após 2 meses de estocagem as castanhas foram consideradas inadequadas para consumo, pelo fato das castanhas estarem em pedaços, o que acelerou as reações de degradação, devido a passagem gradual de oxigênio pela embalagem 
RIBEIRO, S. R.; BOTH, V. e WAGNER, R.

durante armazenamento. Ou seja, era necessário que a embalagem proporcionasse uma barreira maior à passagem de $\mathrm{O}_{2}$ para melhor manutenção da qualidade.

Em um estudo com nozes-pecã armazenadas a temperatura ambiente por 150 dias em filme de náilon-polietileno sob vácuo ou em recipientes plásticos de polipropileno, os resultados indicaram que o teor de umidade não sofreu mudanças significativas (ORO, 2007). Entretanto, foi observado escurecimento da superfície das nozes e aumento significativo nos valores de acidez e peróxidos do óleo extraído ao final do armazenamento $(\mathrm{p}>0,05)$ nas nozes armazenadas em recipientes plásticos. A qualidade das nozes do ponto de vista microbiológica foi considero adequado, com ausência de Salmonella sp. Na análise sensorial não foi percebida redução da aceitabilidade, sendo viável o armazenamento nessas embalagens por 120 dias, não observando diferenças entre as nozes estocadas nos dois tipos de embalagens (ORO, 2007).

\section{Selamento a vácuo}

A aplicação de vácuo na embalagem é uma tecnologia eficaz no armazenamento e comercialização dos produtos (NOSEDA et al., 2012), pois retirando o oxigênio do headspace da embalagem retarda as reações de oxidação e crescimento de microrganismos aeróbios (FLOROS; MATSOS, 2005). Este método não pode ser aplicado a alimentos sensíveis como batata frita e algumas frutas, entretanto tem sido recomendado para nozes, as quais são robustas o suficiente para suportar a compressão. O uso do vácuo também exige embalagens apropriadas, que resultem em baixa troca gasosa. Isso evita que o as nozes alterem o peso e a descoloração. Além disso, devem apresentar alta resistência mecânica (manuseio e transporte), soldabilidade, boa maquinabilidade e custo compatível com a aplicação (HEROBETTA, 2007). Adicionalmente, o emprego do vácuo apresenta ainda como vantagem a redução de tamanho das embalagens dos produtos, o que favorece o transporte (HEROBETTA, 2007).

O armazenamento de amêndoas doces em embalagem a vácuo retardou o aumento do índice de peróxidos por de 7 meses (RAISI, et al., 2015). Ozturk et al., (2016) observaram maior eficiência frente as deteriorações oxidativas quando comparado às demais embalagens com atmosfera modificada em pistache armazenados sob vácuo no período de 30 dias. Por outro lado, Maskan e Karatas (1998) verificaram que tratamento com uso de alto $\mathrm{CO}_{2}(98 \%)$ em pistaches foi mais eficaz que a embalagem a vácuo em relação à oxidação lipídica.

\section{Embalagem com atmosfera modificada}

A demanda o ano todo por produtos em sua apresentação natural e fresca, sem adição de substâncias químicas cresceu consideravelmente. Assim, embalagens com atmosfera modificada (AM) ganharam significativo espaço na indústria alimentícia (FLOROS; MATSOS, 
RIBEIRO, S. R.; BOTH, V. e WAGNER, R.

2005). As modificações das atmosferas das embalagens envolvem normalmente a redução das pressões parciais de oxigênio $\left(\mathrm{pO}_{2}\right)$ e aumento das pressões parciais de dióxido de carbono $\left(\mathrm{pCO}_{2}\right)$ e nitrogênio $\left(\mathrm{pN}_{2}\right)$, sendo controladas apenas no momento de fechar a embalagem. Essas modificações visam estender a vida-de-prateleira dos produtos significantemente, pela manutenção de suas características (FLOROS; MATSOS, 2005). Alguns estudos mostram que a embalagem com AM pode estender em até 10 vezes a vida-de-prateleira de um alimento. Nozes, amêndoas, avelãs e castanhas são exemplos típicos desses alimentos que podem ser beneficiados positivamente com essa técnica (KILCAST; SUBRAMANIAM, 2000), devido ao seu alto teor lipídico, como mencionado anteriormente.

Pistache armazenado em embalagens com ar $\left(\mathrm{pO}_{2}\right.$ de $\left.20 \mathrm{kPa}\right)$, vácuo e $\mathrm{AM}(98 \%$ de $\mathrm{CO}_{2}$ ), em temperatura ambiente apresentaram diferenças significativas nos valores de peróxidos e aumento da acidez somente a partir do terceiro mês de armazenamento. A atmosfera modificada com 98\% de $\mathrm{CO}_{2}$ demonstrou valores menores nos parâmetros de oxidação, podendo ser atribuído à solubilidade do gás na porção líquida e lipídica do pistache, agindo como uma barreira na interface óleo/água diminuindo a ação do oxigênio (MASKAN; KARATAS, 1998). Além disso, essa tecnologia promove a redução da taxa de respiração e o crescimento microbiológico, bem como as alterações enzimáticas, conforme Mazza e Jayas (2001). Entretanto, essa técnica pode apresentar resultados variáveis quanto à qualidade, pois como mencionado anteriormente, o controle dos gases ocorre apenas na instalação da atmosfera. Durante o período de armazenamento, a taxa respiratória do produto, a temperatura de armazenamento, a permeabilidade e seletividade do filme aos gases, o período de armazenamento, entre outro, irão influenciar na efetividade da técnica sobre a manutenção da qualidade.

\section{Atmosfera Controlada}

$\mathrm{O}$ armazenamento em atmosfera controlada (AC) visa prolongar a vida útil de diversas frutas, no entanto é pouco explorada em nuts (RIBEIRO et al., 2020). Na tabela 1 . Essa técnica tem por principal função reduzir a respiração, produção de etileno e reações bioquímicas associadas às alterações fisiológicas (BRACKMANN et al., 2001). No armazenamento em AC os produtos são colocados em um ambiente hermético, onde a $\mathrm{pO}_{2}$ é reduzida e o $\mathrm{pCO}_{2}$ pode ser adicionado/aumentado em relação à condição ambiente. Essa técnica se diferencia da AM pelo fato de ter ajustadas as tensões dos gases com certa frequência, conforme a necessidade do fruto em questão. Além disso, a AC normalmente é associada com o emprego de baixas temperaturas e controle da umidade relativa do ar, potencializando sua eficiência na 
RIBEIRO, S. R.; BOTH, V. e WAGNER, R.

preservação dos alimentos. A tolerância do fruto para a AC depende de vários fatores, incluindo a cultivar, maturidade, pressões parciais dos gases $\left(\mathrm{pO}_{2}, \mathrm{pCO}_{2}\right.$ e $\left.\mathrm{pN}_{2}\right)$ utilizadas na atmosfera e o período de aplicação (BULENS et al., 2012; BAI, et al., 2005). 
Tabela 1. Valores encontrados para índice de acidez e peróxidos em nozes de diferentes espécies após armazenamento por longo período em diferentes condições ou tratamento com irradiação

\begin{tabular}{|c|c|c|c|c|c|c|}
\hline Nut & Método & Tempo & Temperatura & $\begin{array}{c}\text { Indice de } \\
\text { Acidez }\end{array}$ & $\begin{array}{l}\text { Índice de } \\
\text { Peróxidos }\end{array}$ & Referências \\
\hline \multirow[t]{3}{*}{ Amêndoa doce } & Embalagem de PET/LDPE & 12 meses & Ambiente $\left(\sim 25^{\circ} \mathrm{C}\right)$ & - & $>10 \pm 0,01$ & (MEXIS et al., 2009) \\
\hline & $\mathrm{AM}-\mathrm{CO}_{2}(95 \%)$ & 10 meses & $4{ }^{\circ} \mathrm{C}$ & - & $0,75 \pm 0,04$ & (RAISI et al., 2015) \\
\hline & $\mathrm{AM}-\mathrm{CO}_{2}(95 \%)$ & 10 meses & Ambiente $\left(\sim 25^{\circ} \mathrm{C}\right)$ & - & $1,11 \pm 0,07$ & (RAISI et al., 2015) \\
\hline \multirow[t]{2}{*}{ Avelã } & $\mathrm{AM}-\mathrm{N}_{2}(99 \%)$ & 12 meses & $4{ }^{\circ} \mathrm{C}$ & $0,13 \pm 0,01$ & $0,06 \pm 0,03$ & (GHIRARDELLO et al., 2013) \\
\hline & Embalagem plástica & 12 meses & $4{ }^{\circ} \mathrm{C}$ & $0,27 \pm 0,01$ & $0,09 \pm 0,02$ & (GHIRARDELLO et al., 2013) \\
\hline Canarium & Vácuo & 11 meses & Ambiente $\left(22\right.$ a $\left.30^{\circ} \mathrm{C}\right)$ & $0,31 \pm 0,01$ & $0,61 \pm 0,02$ & (WALTON et al., 2017) \\
\hline Macadâmia & Embalagem plástica & 16 meses & Ambiente $\left(20^{\circ} \mathrm{C}\right)$ & - & $3,00 \pm 0,3$ & (HIMSTEDT, 2002) \\
\hline \multirow[t]{3}{*}{ Pecã } & $\mathrm{AC}-3 \mathrm{kPa}$ de $\mathrm{O}_{2}$ & 12 meses & $1^{\circ} \mathrm{C}$ & $0,41 \pm 0,07$ & $1,74 \pm 0,24$ & (RIBEIRO et al., 2020) \\
\hline & $\mathrm{AC}-3 \mathrm{kPa}$ de $\mathrm{O}_{2}$ & 12 meses & $10^{\circ} \mathrm{C}$ & $0,48 \pm 0,01$ & $1,60 \pm 0,01$ & (RIBEIRO et al., 2020) \\
\hline & $\mathrm{AC}-3 \mathrm{kPa}$ de $\mathrm{O}_{2}$ & 12 meses & $20^{\circ} \mathrm{C}$ & $0,62 \pm 0,03$ & $1,55 \pm 0,37$ & (RIBEIRO et al., 2020) \\
\hline \multirow[t]{3}{*}{ Pistache } & 7 kGy & - & - & - & $4,00 \pm 0,05$ & (MEXIS; KONTOMINAS, 2009) \\
\hline & 1 kGy & - & - & $0,18 \pm 0,01$ & $1,01 \pm 0,02$ & (GECGEL et al., 2011) \\
\hline & 7 kGy & - & - & $0,23 \pm 0,05$ & $1,80 \pm 0,01$ & (GECGEL et al., 2011) \\
\hline \multirow[t]{2}{*}{ Walnut } & 1 kGy & - & - & $0,22 \pm 0,01$ & $1,20 \pm 0,5$ & (GECGEL et al., 2011) \\
\hline & 7 kGy & - & - & $0,26 \pm 0,01$ & $1,80 \pm 0,2$ & (GECGEL et al., 2011) \\
\hline
\end{tabular}


As concentrações dos gases da AC necessitam ser ajustados visando manter por maior tempo possível os atributos iniciais dos produtos. Dependendo do produto armazenado, valores elevados de $\mathrm{pCO}_{2}$ podem causar distúrbios fisiológicos, depreciando a qualidade final. Por outro lado, o $\mathrm{O}_{2}$ deve ser mantido em uma pressão mais próxima possível da condição ótima. Concentrações acima da ótima podem não apresentar o efeito desejado, enquanto que aquelas excessivamente baixas podem induzir ao metabolismo fermentativo (THEWES et al., 2015). Ademais, juntamente com temperaturas baixas, as atividades como reações bioquímicas, respiração e oxidação são reduzidas (RIBEIRO et al., 2020; MESSINA, 2015). Outro benefício da $\mathrm{AC}$ é ser uma alternativa contra ataques de insetos, onde fumigantes químicos não são permitidos (SEM et al., 2010).

Pecãs armazenadas em casca a 1,10 e $20^{\circ} \mathrm{C}$ e em AC de $1 \mathrm{kPa}, 3 \mathrm{kPa}$ de $\mathrm{O}_{2}$ e atmosfera de ambiente $\left(20 \mathrm{kPa}\right.$ de $\left.\mathrm{O}_{2}\right)$ por até 12 meses apresentaram pouca diferença nas condições com AC de menor temperatura e pressão de oxigênio, para parâmetros de oxidação avaliados (cor, acidez, peróxidos). Por outro lado, ocorreu um aumento significativo na maior condição de $\mathrm{pO}_{2}$ (ambiente) e temperatura de $20{ }^{\circ} \mathrm{C}$ (RIBEIRO, et al., 2020). No trabalho de Guadagni (1978) com amêndoas doces armazenadas em casca em AC, o armazenamento foi eficaz contra deteriorações e formação de sabores rançosos pelo período de 12 meses mantidos em temperatura ambiente $\left(18^{\circ} \mathrm{C}-27^{\circ} \mathrm{C}\right)$. Em AC, as câmaras de armazenamento são fechadas por longos períodos e o desenvolvimento microbiano anormal não pode ser detectado visualmente e, quando observadas, muitas vezes o estágio avançado das modificações do metabolismo do fruto não permite intervenções (LÓPEZ et al., 2015). Dessa forma, a recomendação é que seja feita uma amostragem periódica para a avaliação dos produtos que estão armazenados nas câmaras, especialmente em produtos em que não existem muitos estudos realizados ainda, a exemplo da noz-pecã.

\section{Considerações Finais}

Esta revisão foi realizada visando estudar os diferentes tipos de técnicas e embalagens que podem ser utilizadas para manter a qualidade no armazenamento de nozes, amêndoas e castanhas, com atenção especial para a noz-pecã. Com a quebra da casca ou descascamento total, as amêndoas das nozes ficam expostas diretamente ao ar, facilitando as degradações oxidativas e contaminações microbianas. Sendo assim, a determinação das condições ideais e a seleção adequada dos métodos de armazenamento, bem como as embalagens tornam-se necessárias para evitar a absorção de umidade, retardar o crescimento microbiano, evitar a 
RIBEIRO, S. R.; BOTH, V. e WAGNER, R.

formação de peróxidos, hidrólise e oxidação lipídica, escurecimento, entre outros, que influenciam na qualidade e segurança geral da nut armazenada.

Apesar de não haver muita pesquisa com o armazenamento da noz-pecã, alguns aspectos foram reportados em relação à temperatura e pressões parciais de gases no armazenamento. Certamente ainda existem muitas lacunas no conhecimento que necessitam ser preenchidas com relação à manutenção da qualidade pós-colheita desta cultura. Estes estudos podem ser conduzidos a partir dos resultados obtidos em outras nuts, por isso a importância de abordar também estes aspectos nesta revisão. Com a tendência de aumento na produção nacional de pecã, em função do aumento significativo da área de plantio da cultura, aliado à colheita concentrada em um curto período do ano (abril e maio), certamente o tema armazenamento será uma grande demanda do setor, em busca da manutenção da qualidade. Isso justifica a sequência dos trabalhos visando ofertar noz-pecã de qualidade aos consumidores durante o ano todo.

\section{Referências}

AGENCIA NACIONAL DE VIGILÂNCIA SANITÁRIA. Resolução da diretoria colegiadaResolução-RDC $\mathbf{N}^{\mathbf{0}}$ 270, de 22 de Setembro de 2005. Disponível em:< http://bvsms.saude.gov.br/bvs/saudelegis/anvisa/2005/rdc0270_22_09_2005.html> Acessado em: 08 jul. 2020.

ALGABR, H. M.; ZHENG, T.; YU, X. 2013. Inactivation of Aspergillus flavus in drinking water after treatment with UV irradiation followed by chlorination. Science of the Total Environment, v. 525:9, p. 463 - 464. https://doi.org/10.1016/j.scitotenv.2013.06.065

ASSOCIAÇÃO BRASILEIRA DE NOZES, CASTANHAS E FRUTAS SECAS, ABNC. Brasil atinge a marca de $4^{\circ}$ maior produtor mundial de noz - pecã. Disponível em: http://www.abncnuts.org.br/ Acesso em 24 de junho de 2020

ATSU BARKU, V. Y.; NYARKO, H. D.; DORDUNU, P. 2012, Studies on the physicochemical characteristics, microbial load and storage stability of oil from indian almond nut (Terminalia catappa L.), Food Science and Quality Management, v. 8:1, p. 9-17, 2012. https://doi.org/10.12691/ajfst-3-4-1

BAI, J.; BALDWIN, E. A.; GOODNER, K. L.; MATTHEIS, J. P.; BRECHT, J. K. 2005 Response of four apple cultivars to 1-methylcyclopropene treatment and controlled atmosphere storage, Horticultural Science, v. 40, p. 1534-1538, 2005. https://doi.org/10.21273/HORTSCI.40.5.1534

BARREIRA, J. C. M.; ANTONIO, A. L.; GÜNAYDI, T.; ALKAN, H.; BENTO, A.; BOTELHO, L, M.; FERREIRA, I. C. F. R. 2012, Chemometric characterization of gamma irradiated chestnuts from Turkey. Radiation Physics and Chemistry, v. 81:9, p. 1520-1524. https://doi.org/10.1016/j.radphyschem.2012.01.005

BOUKAEW, S.; PRASERTSAN, P.; SATTAYASAMITSATHIT, S.; 2017. Evaluation of antifungal activity of essential oils against aflatoxigenic Aspergillus flavus and their 
allelopathic activity from fumigation to protect maize seeds during storage. Industrial Crops and Products. v. 97, p. 558-566. https://doi.org/10.1016/j.indcrop.2017.01.005

BRACKMANN, A.; MELLO, A. M.; FREITAS, S. T.; VIZZOTO, M.; STEFFENS, C. A. 2001 Armazenamento de maçãs 'Royal Gala' sob diferentes temperaturas e pressões parciais de oxigênio e gás carbônico. Revista Brasileira de Fruticultura, Jaboticabal, v. 23:3, p. 532536, 2001. https://doi.org/10.1590/S0100-29452001000300016

BULENS, I.; VAN DE POEL, B.; HERTOG, M.L.A.T.M.; DE PROFT, M.P.; GEERAERD, A.H.; NICOLAI, B.M.; 2012 Influence of harvest time and 1-MCP application on postharvest ripening and ethylene biosynthesis of 'Jonagold' apple, Postharvest Biology Technology, v.72, p. 11-19, 2012. https://doi.org/10.1016/j.postharvbio.2012.05.002

CARGNELUTTI-FILHO, A.; POLETTO, T.; MUNIZ, M. F. B.; BAGGIOTTO, C.; POLETTO, I. 2014 Dimensionamento amostral para avaliação da massa e diâmetro de frutos de nogueira-pecã, Ciência Rural, ISSN 0103-8478, 2014. http://dx.doi.org/10.1590/0103$\underline{8478 \mathrm{cr} 20140964}$

DE MELLO, F. R.; SCUSSEL, V. M. 2009 Development of physical and optical methods for in-shell Brazil nuts sorting and aflatoxin reduction, Journal of Agricultural Science, v. 1, 314, 2009. https://doi.org/10.5539/jas.v1n2p3

ERTEN, E.S.; Characterization of aroma components of raw and roasted almonds and the effect of the oxidative state of the nut on roasted flavor formation, Thesis (Doctorate in Philosophy of Food Science and Human Nutrition), University of Illinois at UrbanaChampaign, Urbana, Illinois, 2016.

FANG, Y.; HU, J.; XIONG, S.; ZHAO, S. 2011 Effect of low-dose microwave radiation on Aspergillus parasiticus Food Control, v. 22, p. 1078-1084, 2011. https://doi.org/10.1016/j.foodcont.2011.01.004

FLOROS, J. D.; MATSOS, K. L. 2005 Innovations in food packaging. Elsevier, Academic Press, Cap. 10, p. 159-171, 2005

FRONZA, D.; HAMANN, J. J.; Técnicas para o cultivo da nogueira-pecã. Santa Maria: Universidade Federal de Santa Maria, Colégio Politécnico, Núcleo de Fruticultura Irrigada, 2016.

FRONZA, D.; HAMANN, J. J.; BOTH, V.; ANESE, R. O.; MEYER, E. A. 2018 Pecan cultivation: general aspects. Ciência Rural, v. 48:2, 2018. https://doi.org/10.1590/0103$\underline{8478 \mathrm{cr} 20170179}$

FRONZA, D.; POLETTO, T.; HAMANN, J. J.; O cultivo da nogueira-pecã. Santa Maria: Universidade Federal de Santa Maria, Colégio Politécnico, Núcleo de Fruticultura Irrigada, 2015.

GAMA, T.; WALLACE, H. M.; TRUEMAN, S. J.; HOSSEINI-BAI, S. 2018. Quality and shelf life of tree nuts: A review. Scientia Horticulturae, v. 242, p. 116-126. https://doi.org/10.1016/j.scienta.2018.07.036 
GARCÍA-PASCUAL, P.; MATEOS, M.; CARBONELL, V.; SALAZAR, D. M. 2003 Influence of storage conditions on the quality of shelled and roasted almonds. Biosystems Engineering, v. 84:2, p. 201-209, 2003. https://doi.org/10.1016/S1537-5110(02)00262-3

GECGEL, U.; GUMUS, T.; TASAN, M.; DAGLIOGLU, O.; ARICI, M. 2011. Determination of fatty acid composition of $\gamma$-irradiated hazelnuts, walnuts, almonds, and pistachios. Radiation $\begin{array}{llllll}\text { Physics and Chemistry, } & \text { v. 80:4, p. }\end{array}$ https://doi.org/10.1016/j.radphyschem.2010.12.004

GHIRARDELLO, D.; CONTESSA, C.; VALENTINI, N.; ZEPPA, G.; ROLLE, L.; GERBI, V., BOTTA, R. 2013 Effect of storage conditions on chemical and physical characteristics of hazelnut (Corylus avellana L.). Postharvest Biology and Technology, v. 81, p. 37-43, 2013. https://doi.org/10.1016/j.postharvbio.2013.02.014

GUADAGNI, D. G.; SODERSTROM, E. L.; STOREY, C. L. 1978, Effects of controlled atmosphere on flavor stability of almonds. Journal of Food Science, v. 43:4, p. 1077-1080, 1978. https://doi.org/10.1111/j.1365-2621.1978.tb15237.x

HEROBETTA, D. 2006 Aumente o tempo de prateleira de seus produtos com embalagens a vácuo. Revista Frigorífico, 2006. Edição 130. Disponível em: http://www.revistafrigorifico.com.br/revista/edicao_130_vacuo.htm. Acesso em: 10 jun. 2020.

IVANOVA-PETROPULOS, V.; MITREV, S.; STAFILOV, T.; MARKOVA, N.; LEITNER, E., LANKMAYR, E.; SIEGMUND, B. 2015 Characterisation of traditional Macedonian edible oils by their fatty acid composition and their volatile compounds. Food Research International, v. 77:3, p. 506-514, 2015. https://doi.org/10.1016/j.foodres.2015.08.014

KAYA, A.; TEKIN, A. R; ONER, M. D. 1993 Oxidative stability of sunflower and olive oils: comparison between a modified active oxygen method and long term storage. Lebensmittel Wissenchat Techonologie, v. 26:5, p. 464-468, 1993. https://doi.org/10.1006/fstl.1993.1091

KARAGÖZ, I.; MOREIRA, R. G.; CASTELL-PEREZ, M. E. 2014 Radiation D10 values for Salmonella Typhimurium LT2 and an Escherichia coli cocktail in pecan nuts (Kanza cultivar) exposed to different atmospheres, Food Control, v. 39, pp. 146 - 1532014. https://doi.org/10.1016/j.foodcont.2013.10.041

KILCAST, D.; SUBRAMANIAM, P. 2000 The stability and shelf-life of food. Cap 7. Woodhead Publishing Limited. Elsevier, Cambridge, London, 2000, ISBN 978-0-08-1004357, https://doi.org/10.1016/C2015-0-06842-3

KING, J. C.; BLUMBERG, J.; INGWERSEN, L.; JENAB, M.; TUCKER, K. L. 2008 Tree nuts and peanuts as components of a healthy diet. The Journal of Nutrition, v. 138, p. $1736-1740$, 2008. https://doi.org/10.1093/jn/138.9.1736S

KUME, T.; FURUTA, M.; TODORIKI, S.; UENOYAMA, N.; KOBAYASHI, Y. 2009. Status of food irradiation in the world. Radiation Physics and Chemistry. v. 78, p. 222-226, 2009. https://doi.org/10.1016/j.radphyschem.2012.01.005

LEE, H.; RYU, J.; KIM, H. 2020. Antimicrobial activity of gaseous chlorine dioxide against Aspergillus flavus on green coffee beans, v. 86, 103308, 2020. https://doi.org/10.1016/j.fm.2019.103308 
LESINA, E. Cultivo da noz-pecã se expande e ganha espaço no Rio Grande do Sul. Jornal do comércio - Agronegócios. Edição impressa de 10/02/2020. Disponível em: https://www.jornaldocomercio.com/_conteudo/economia/2020/02/724121-cultivo-da-nozpeca-se-expande-eganha-espaco-no-rio-grande-do-sul.html (2020) Acesso em: 21 mar. 2020.

LÓPEZ, L.; ECHEVERRIA, G.; USALL, J.; TEIXIDÓ, N. 2015 The detection of fungal diseases in the 'Golden Smoothee' apple and 'Blanquilla' pear based on the volatile profile. Postharvest Biology and Technology, v. 99, p. 120-130, 2015. https://doi.org/10.1016/j.postharvbio.2014.08.005

LUTER, L.; WYZLOUZIL, W.; KASHYAP, S. C. 1982 The destruction of aflatoxins in peanuts by microwave roasting, Canadian Institute of Food Science and Technology Journal, v. 15, p. 234-238, 1982. https://doi.org/10.1016/S0315-5463(82)72545-3

MA, Y.; LU, X.; LIU, X.; MA,. H.; 2013 Effect of ${ }^{60}$ Co $\gamma$-irradiation doses on nutrients and sensory quality of fresh walnuts during storage, Postharvest Biology and Technology, v. 84, 36-42, 2013. https://doi.org/10.1016/j.postharvbio.2013.04.001

MA, Y.; WANG, G. L.; LIU, X.; LI, Y. C. 2010 Germination and sprouting physiology of fresh walnut irradiated by ${ }^{60} \mathrm{Co} \gamma$-irradiation. Acta Bot. Boreali-Occident. v. 30:10, p. 2034-2039, 2010. https://doi.org/10.1016/j.postharvbio.2013.04.001

MASKAN, M.; KARATAS, S. 1998 Fatty acid oxidation of pistachio nuts stored under various atmospheric conditions and different temperatures. Journal of Science and Food Agriculture, v. 77, p. 334-340, 1998. https://doi.org/10.1002/(SICI)1097-0010(199807)77:3<334::AIDJSFA42>3.0.CO;2-A

MAZZA, G.; JAYAS, D. S. 2001 Controlled and Modified Atmosphere Storage. In: ESKIN, N. A. M.; ROBINSON, D. S. (Eds.) Food Shelf Life Stability - Chemical, Biochemical and Microbiological Changes. CRC Press LLC, Boca Raton, 2001.

MESSINA, C. M.; BONO, G.; RENDA, G.; BARBERA, L. L.; SANTULLI, A. 2015 Effect of natural antioxidants and modified atmosphere packaging in preventing lipid oxidation and increasing the shelf-life of common dolphinfish (Coryphaena hippurus) fillets, LWT - Food Science and Technology, v. 62:1, p. 271-277, 2015. https://doi.org/10.1016/j.lwt.2015.01.029

MEXIS, S. F.; BADEKA, A. V.; KONTOMINAS, M. G. 2009. Quality evaluation of raw ground almond kernels (Prunus dulcis): Effect of active and modified atmosphere packaging, container oxygen barrier and storage conditions. Innovative Food Science \& Emerging Technologies, v. 10:4, 580-589, 2009 https://doi.org/10.1016/j.ifset.2009.05.002

MEXIS, S. F.; KONTOMINAS, M. G. 2009a Effect of $\gamma$-irradiation on the physicochemical and sensory properties of cashew nuts (Anacardium occidentale L.). LWT - Food Science and Technology v. 42:9, p. 1501-1507, 2009a. https://doi.org/10.1016/j.lwt.2009.03.023

MEXIS, S. F.; KONTOMINAS, M. G. 2009b Effect of gamma irradiation on the physicochemical and sensory properties of raw shelled peanuts (Arachis hypogaea L.) and pistachio nuts (Pistacia vera L.), Journal of the science of Food anda Agriculture, v. 89:5, p. 867-875, 2009b. https://doi.org/10.1002/jsfa.3526 
MIN, D. B.; BOFF, J. M. 2002. Chemistry and reaction of singlet oxygen in foods Comprehensive Reviews in Food Science and Food Safety, v. 1, p. 58-72, 2002 https://doi.org/10.1111/j.1541-4337.2002.tb00007.x

MOKOCHINSKI, F. M. Estimativa de produção, caracterização física e perfil químico de amêndoas de nogueira-pecã; Dissertação (Mestrado em Agronomia), Universidade Estadual do Centro-Oeste, UECO, Guarapuava - Paraná, p. 17, 2015.

MURDOCK, D. H. Encyclopedia of foods: a guide to healthy nutrition. San Diego: Academic Press, p. 529, 2002.

NOSEDA, B.; ISLAM, M.T.; ERIKSSON, M.; HEYNDRICKX, M.; DE REU, K.; VAN LANGENHOVE, H.; DEVLIEGHERE, F.; 2012 Microbiological spoilage of vacuum and modified atmosphere packaged Vietnamese Pangasius hypophthalmus fillets. Food Microbiology, v. 30, p. 408-419, 2012. https://doi.org/10.1016/j.fm.2011.12.025

OGUNSINA B. S.; BAMGBOYE A. I. 2012 Effect of Moisture Content, Nut Size and Hot-Oil Roasting Time on the Whole Kernel "Out-Turn" of Cashew Nuts (Anacardium occidentale) During Shelling. Nigerian Food Journal, v. 30:2, p. 57-65, 2012. https://doi.org/10.1016/S0189-7241(15)30036-9

ORO, T.; OGLIARI, P. J.; AMBONI, R. D. M. C.; BARRERA-ARELLANO, D.; BLOCK, J. M. 2008 Evaluación de la calidad durante el almacenamiento de nueces Pecán [Carya illinoinensis (Wangenh.) C. Koch] acondicionadas en diferentes envases. Grasas y Aceites, v. 59:2, p. 132-138, 2008. https://doi.org/10.3989/gya.2008.v59.i2.501

ORO, T.; Composição nutricional, compostos bioativos e vida útil de noz e óleo extravirgem de noz-pecã [Carya illinoinensis (Wangenh) C. Koch] 2007. 105 p. Dissertação (Mestrado em Ciência dos Alimentos) - Centro de Ciências Agrárias, Universidade Federal de Santa Catarina, Florianópolis, 2007.

OZTURK, I.; SAGDIC, O.; YALCIN, H.; CAPAR, T. D.; ASYALI, M. H. 2016. The effects of packaging type on the quality characteristics of fresh raw pistachios (Pistacia vera L.) during the storage. LWT - Food Science and Technology., 65, 457-463. https://doi.org/10.1016/j.lwt.2015.08.046

PANKAEW, P.; JANJAI, S.; NILNONT, W.; PHUSAMPAO, C.; BALA, B.K. 2016. Moisture desorption isotherm, diffusivity and finite element simulation of drying of macadamia nut (Macadamia integrifolia). Food and Bioproducts Processing, v. 100, p. 16-24, 2016.

RAEI, M.; MORTAZAVI, A.; POURAZARANG, H. 2009 Effects of Packaging Materials, Modified Atmospheric Conditions, and Storage Temperature on Physicochemical Properties of Roasted Pistachio Nut. Food Analytical Methods, v. 3:2, p. 129-132, 2009. https://doi.org/10.1007/s12161-009-9076-1

RAINEY, C.; NYQUIST, L.; Nuts: nutrition and health benefits of daily use. Nutrition Today, v. 32:4, p. 157-163, $1997 . \quad$ Disponível em: https://journals.lww.com/nutritiontodayonline/Abstract/1997/07000/Nuts_Nutrition_and_Heal th_Benefits_of_Daily_Use.6.aspx Acesso em: 05 jul 2020

RAISI, M.; GHORBANI, M.; MAHOONAK, A. S.; KASHANINEJAD, M.; HOSSEINI, H. 2015 Effect of storage atmosphere and temperature on the oxidative stability of almond kernels 
RIBEIRO, S. R.; BOTH, V. e WAGNER, R.

during long term storage, Journal of Stored Products Research, v. 62, p. 16-21, 2015. https://doi.org/10.1016/j.jspr.2015.03.004

RIBEIRO, M. A. A.; REGITANO-D’ARCE, M. A. B.; LIMA, U. A.; NOGUEIRA, M. C. S. 1993 Storage of canned shelled Brazil nuts: effects on the quality. Acta Alimentaria, v. 22:4, p. 295-303, 1993.

RIBEIRO, S. R.; RIBEIRO, Q. M.; KLEIN, B.; SANTOS, I. D.; FORGIARINI, S.; HAMANN, J. J.; CICHOSKI, A. J.; FRONZA, D.; BRACKMANN, A.; BOTH, V.; WAGNER, R. 2020 Effect of low oxygen on quality attributes of 'Barton' pecan nuts after long-term storage at different temperatures, Scientia Horticulturae, v. 263, 109098, 2020. https://doi.org/10.1016/j.scienta.2019.109098

SAHENA, F.; ZAIDUL, I. S. M.; JINAP, S.; KARIM, A. A.; ABBAS, K. A.; NORULAINI, N. A. N.; OMAR A. K. M. 2009 Application of supercritical $\mathrm{CO}_{2}$ in lipid extraction - A review. $\begin{array}{llllllll}\text { Journal of Food Engineering, v. } & \text { 95: } 2009 .\end{array}$ https://doi.org/10.1016/j.jfoodeng.2009.06.026

SALVADOR, A. A. Atividade antioxidante e perfil de ácidos graxos de extratos da torta de noz pecã [Carya illinoensis (Wangenh) K. Koch] obtidos por extração supercrítica, Dissertação (Mestrado em Engenharia de Alimentos), Centro Tecnológico da Universidade Federal de Santa Catarina, Florianópolis, Santa Catarina 2014.

SALVADOR, A. A.; PODESTÁ, R.; BLOCK, J. M.; FERREIRA, S. R. S. 2016 Increasing the value of pecan nut [Carya illinoinensis (Wangenh) C. Koch] cake by means of oil extraction and antioxidant activity evaluation. Journal. of Supercritical Fluids, v. 116, p. 215-222, 2016. https://doi.org/10.1016/j.supflu.2016.05.046

SANTERRE, C. R. Pecan Technology. New York: Chapman \& Hall: 161 p. 1994.

SATTAR, A.; JAN, M.; AHMAD, A.; HUSSAIN, A.; KHAN, I. 1989 Light induced oxidation of nut oils (Short communication). Food Nahrung v. 33:2, p. 213-215, 1989. https://doi.org/10.1002/food.19890330226

SEM, F.; MEYVACI, K. B.; TURANLIB, F.; AKSOY, U.; 2010 Effects of short-term controlled atmosphere treatment at elevated temperature on dried fig fruit, Journal of Stored Products Research; v. 46, p. 28-33, 2010. https://doi.org/10.1016/j.jspr.2009.07.005

SILVA, A. C.; SARTURI, H. J.; DALl'OGLIO, E. L.; SOARES, M. A.; SOUSA, P. T.; VASCONCELOS, L. G.; KUHNEN, C. A. 2016. Microwave drying and disinfestation of Brazil $\begin{array}{lllllll}\text { nut seeds. } & \text { Food } & \text { Control, } & \text { v. } & 70, & \text { p. } & 119-129,\end{array}$ https://doi.org/10.1016/j.foodcont.2016.04.049

SILVA, C. 2017 Produtores comemoram boa safra de noz-pecã - Jornal do Comércio, Porto Alegre, $08 \quad$ mai. 2017.20 Disponível em http://jcrs.uol.com.br/_conteudo/2017/04/economia/559613-produtores-comemoram-boasafra-de-noz-peca.html Acesso em:15 mar. 2020

SOHBATZADEH, F.; MIRZANEJHAD, S.; SHOKRI, H.; NIKPOUR, M. 2016. Inactivation of Aspergillus flavus spores in a sealed package by cold plasma streamers. Journal of Theoretical and Applied Physics, v. 10, p. 99-106. https://doi.org/10.1007/s40094-016-0206$\underline{\mathrm{Z}}$ 
THEWES, F. R.; BOTH, V.; BRACKMANN, A.; WEBER, A.; ANESE, R. O. 2015. Dynamic controlled atmosphere and ultralow oxygen storage on "Gala" mutants quality maintenance. Food Chemistry, v. 188, p. 62-70, 2015. https://doi.org/10.1016/j.foodchem.2015.04.128

THOMPSON, J. F.; KADER, A. A. 2012 Harvesting, transporting, processing and grading, Chapter 17, in: Ferguson, L.; 2012 Pistachio production manual. Oakland, University of California, Agriculture and Natural Resources, 2012.

TORABIAN, S.; HADDAD, E.; RAJARAM, S.; BANTA, J.; SABATÉ, J. 2009. Acute effect of nut consumption on plasma total polyphenols, antioxidant capacity and lipid peroxidation. Journal of Human Nutrition and Dietetics. v. 22, p. 64-71, 2009. https://doi.org/10.1111/j.1365-277X.2008.00923.X

VENKATACHALAM, M.; SATHE, S. K. 2006. Chemical composition of selected edible nut seeds. Journal of Agricultural and Food Chemistry, v. 54:13, p. 4705-4714 2006. https://doi.org/10.1021/jf0606959

VILLARREAL-LOZOYA, J. E.; LOMBARDINI, L.; CISNEROS-ZEVALLOS，L. 2007 Phytochemical constituents and antioxidant capacity of different pecan [Carya illinoinensis (Wangenh.) K. Koch] cultivars; Food Chemistry, v. 102, p. 1241-1249, 2007. https://doi.org/10.1016/j.foodchem.2006.07.024

WALTON, D. A.; RANDALl, B. W.; POIENOU, M.; NEVENIMO, T.; MOXON, J.; WALLACE, H. M. 2017. Shelf Life of Tropical Canarium Nut Stored under Ambient Conditions. Horticulturae v. 3:1, 24, 2017. https://doi.org/10.3390/horticulturae3010024

WOODROOF, J. G.; Pecan history and distribution. In: Tree Nuts: Production, Processing, Products, v. 2, p. 1-38, Westport, Conn: AVI, 1967.

WORLD CANCER RESEARCH FUND (WCRF). Food, nutrition, physical activity, and prevention of cancer: a global perspective. Washington (DC): AICR; 2007. Disponível em: http://www.wcrf.org/int/research-we-fund/continuous-update-project-cup , Acessado em $21 \mathrm{de}$ maio de 2020.

ZHAO, X.; LIU, A.; ZHANG, W.; LV, S.; REN, K. 2011 The effect of pecan oil on hippocampal cells apoptosis in ovariectomized rats; Procedia Engineering, v. 18 p. 375-380, 2011 https://doi.org/10.1016/j.proeng.2011.11.060 